\title{
Surgical outcome of laparoscopic colectomy for colorectal cancer in obese patients: A comparative study with open colectomy
}

\author{
YANTAO CAI ${ }^{*}$, YIMING ZHOU* ${ }^{*}$ ZHENYANG LI, JIANBIN XIANG and ZONGYOU CHEN \\ Department of General Surgery, Huashan Hospital Affiliated to Fudan University, Shanghai 200040, P.R. China
}

Received February 1, 2013; Accepted July 25, 2013

DOI: $10.3892 / \mathrm{ol} .2013 .1508$

\begin{abstract}
The aim of the present study was to assess the short-term outcome and survival time of 166 obese patients who received laparoscopic and open colectomy for colorectal cancer (CRC) between January 2007 and December 2012. All 166 patients included in the study had a BMI >28. Laparoscopic or open colectomy procedures were performed on 64 and 102 patients, respectively. The short-term outcome and post-operative survival rates were compared. The patient characteristics were similar between the two groups. Laparoscopic colectomy correlated with an increased duration of surgery compared with open colectomy (183 vs. $167 \mathrm{~min}$, respectively; $\mathrm{P}<0.05$ ) but intraoperative blood loss was decreased (168 vs. $188 \mathrm{ml}$, respectively; $\mathrm{P}<0.05)$. Hospitalization costs were slightly higher following the laparoscopic procedure compared with open surgery, but this was affordable for the majority of patients ( $¥ 56,484$ vs. $¥ 56,161$, respectively; $\mathrm{P}<0.05$ ). The incidence of wound infection (17 vs. $31 \%$; $\mathrm{P}<0.05)$ and abdominal abscess rates $(6$ vs. $18 \% ; \mathrm{P}<0.05)$ were reduced in the laparoscopic group compared with the open group. Pathological characteristics were identified to be similar and no significant differences were identified in overall (log-rank test; $\mathrm{P}=0.85$ ) and disease-free (log-rank test; $\mathrm{P}=0.85)$ survival between the two types of surgery (log-rank test; $\mathrm{P}=0.76$ ). The current retrospective study demonstrated an improved short-term outcome in laparoscopic colectomy for CRC patients with a $\mathrm{BMI}>28$ compared with patients who underwent the open procedure. Laparoscopic colectomy is technically and oncologically safe and must be popularized in obese CRC patients.
\end{abstract}

Correspondence to: Dr Zongyou Chen or Dr Jianbin Xiang, Department of General Surgery, Huashan Hospital, Fudan University, 12 Wulumuqizhong Road, Shanghai 200040, P.R. China E-mail: zongyouchen@hotmail.com

E-mail: xjbzhw@163.com

* Contributed equally

Key words: colorectal, obesity, laparoscopic, open

\section{Introduction}

Obesity is an increasing social problem with a significant effect on an individuals health that has subsequently been defined as a disease by the World Health Organization (WHO) (1). Due to changing diets and growing socioeconomic prosperity, the prevalence of overweight and obese individuals in China has increased to $29.9 \%$ according to data from the China National Nutrition and Health Survey in 2002 (2). It has been proven that obesity is an independent risk factor associated with an increased comorbidity, post-operative morbidity, risk of anesthesia and difficulties in surgery. The introduction of laparoscopic techniques initiated a new era for colorectal cancer (CRC) surgery. Previous studies have shown that a laparoscopy is less invasive with improved short-term outcomes compared with traditional open surgery (3). The anatomical development of complete mesocolic and total mesorectal excision in colon and rectal cancer, respectively, has improved oncological safety. Previously, obesity prevented the use of laparoscopic colorectal surgery, however, following improvements to the instruments and techniques involved, this procedure may now be performed in obese individuals, although it requires surgical expertise to perform the procedure safely. However, a number of studies have shown inevitably higher rates of conversion and post-operative complications in the laparoscopic surgery of obese patients compared with non-obese patients (4-6).

The present study was conducted to compare the short-term outcome and survival time of obese patients receiving laparoscopic or open colectomy for CRC. The results are likely to aid in the selection of a suitable surgical approach for obese CRC patients.

\section{Materials and methods}

Eligibility. Between January 2007 and December 2012, 166 patients with a pre-operative BMI $>28$ underwent laparoscopic or open colectomy for CRC in the Department of General surgery, Huashan Hospital (Shanghai, China). The exclusion criteria were as follows: i) Patients who had a tumor that could not be resected radically; ii) patients who had undergone abdominoperineal resection; iii) a history of previous gastrointestinal surgical history; iv) patients who had received neoadjuvant chemoradiotherapy prior to surgery; v) patients who had undergone emergency surgery; and vi) cases with 
Table I. Summary of patient characteristics and comparison between the open and laparoscopic groups.

\begin{tabular}{|c|c|c|c|}
\hline & Open & Laparoscopic & P-value \\
\hline Patients, $\mathrm{n}$ & 102 & 64 & \\
\hline Gender, $\mathrm{n}$ & & & 0.502 \\
\hline Male & 60 & 41 & \\
\hline Female & 42 & 23 & \\
\hline Age, years & $63.1 \pm 11.5$ & $64.4 \pm 13.1$ & 0.258 \\
\hline BMI & $29.69 \pm 1.51$ & $29.28 \pm 1.25$ & 0.567 \\
\hline Tumor location, $\mathrm{n}$ & & & 0.429 \\
\hline Cecum & 9 & 7 & \\
\hline Ascending colon & 28 & 13 & \\
\hline Transverse colon & 12 & 5 & \\
\hline Descending colon & 7 & 7 & \\
\hline Sigmoid colon & 33 & 18 & \\
\hline Rectum & 13 & 14 & \\
\hline ASA class, $n$ & & & 0.196 \\
\hline 1 & 36 & 18 & \\
\hline$\geq 2$ & 66 & 46 & \\
\hline \multicolumn{4}{|c|}{ Pre-operative complications, $\mathrm{n}$} \\
\hline Cardiovascular & 48 & 31 & 0.753 \\
\hline Endocrine & 19 & 12 & 0.312 \\
\hline
\end{tabular}

Data are mean \pm standard deviation unless otherwise stated. ASA, American Society of Anethesiologists.

incomplete medical records. This study was approved by the ethics committee of Huashan Hospital Affiliated to Fudan University (Shanghai, China). Written informed consent was obtained from the patient's family.

Data collection methods. Data were obtained from the patient medical record database of Huashan Hospital (Shanghai, China), including: i) Patient characteristics, i.e., gender, age, BMI, tumor location, American Society of Anesthesiologist classification and pre-operative complications; ii) intraoperative data, i.e., operating time, blood loss and conversion to open procedure; iii) post-operative data, i.e., first bowel movement, number of days to initiation of fluid diet, drainage length and the length and cost of hospitalization; and iv) post-operative complications, i.e., wound infection, anastomotic leak, pulmonary complications, abdominal abscess, ileus, chyle leakage, hemorrhage, deep vein thrombosis and repeat surgery. Length of hospitalization was defined as the period between admittance and discharge. Patients were followed at outpatient clinics every 3 months for the first 2 years and every 6 months thereafter, in addition to phone calls, mail and e-mail follow-ups.

Surgical procedure. Patients with pre-operative complications were administered with appropriate therapy prior to surgery under consultation. For the laparoscopic surgery, four or five trocars were inserted whilst pneumoperitonium pressure was maintained at $10-14 \mathrm{mmHg}$. The exact surgical type was determined according to the location of the tumor and via intraoperative detection. The principle of obtaining a radical cure was followed during surgery. Staplers were applied to achieve anastomosis in the two groups and drainage was used routinely at the correct locations. Patients with T3/T4 or lymph node metastasis received post-operative systemic adjuvant chemotherapy according to the National Comprehensive Cancer Network (NCCN) Clinical Practice Guidelines in Rectal Cancer, 2011.

Statistical analysis. The statistical analysis was performed using the SPSS 19.0 statistical software package (SPSS Inc., Chicago, IL, USA). Continuous data are presented as the mean \pm SD or as indicated. Quantitative variables were analyzed by Student's t-test, and $\chi^{2}$ and Fisher's exact tests were used to analyze group comparisons where appropriate. Overall and disease-free survival rates were analyzed using Kaplan-Meier curves and evaluated with the log-rank test. $\mathrm{P}<0.05$ was considered to indicate a statistically significant difference.

\section{Results}

A total of 166 eligible patients who underwent laparoscopic or open colectomy for CRC between 2007 and 2012 were distributed into two groups according to the type of surgery performed. A total of 64 patients formed the laparoscopic group and 102 patients formed the open group. No significant differences in demographic data were identified between the two groups (Table I). 
Table II. Intraoperative data and post-operative complications.

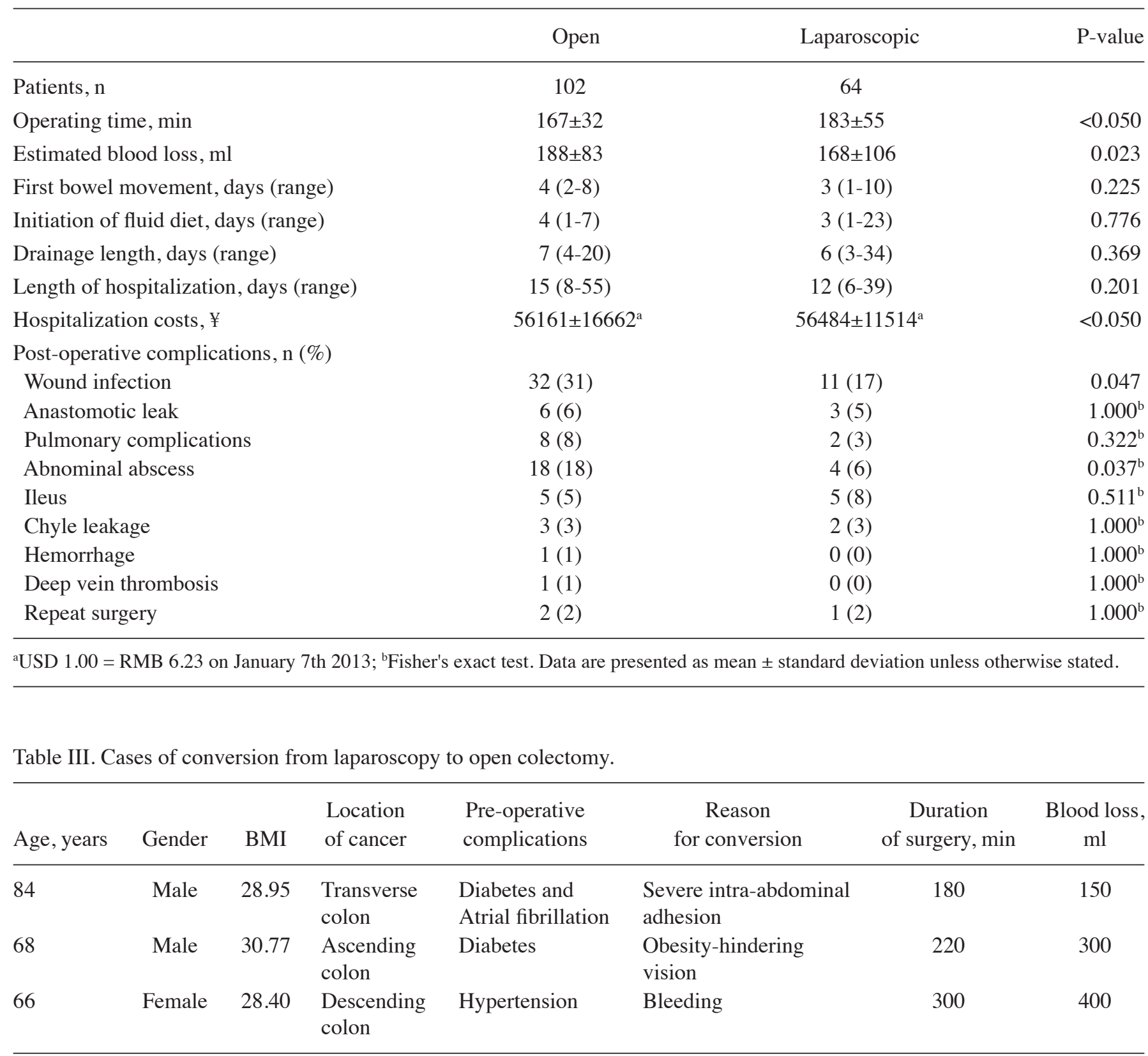

Table IV. Repeat surgery at 30 days.

\begin{tabular}{|c|c|c|c|c|c|}
\hline Age, years & Gender & BMI & Comorbidity & First surgery & Reason for repeat surgery \\
\hline 80 & Male & 29.57 & Hypertension and Diabetes & Open anterior resection & Anastomotic hemorrhage \\
\hline 58 & Male & 30.46 & None & Open right hemicolectomy & Anastomotic leakage \\
\hline 82 & Male & 28.86 & Diabetes & Laparoscopic anterior resection & Small bowel obstruction \\
\hline
\end{tabular}

Table II presents the intra- and post-operative data. The duration of surgery was higher in the laparoscopic group compared with the open group (183 vs. 167 min, respectively; $\mathrm{P}<0.05)$, in addition to a significantly reduced intraoperative blood loss (168 vs. $188 \mathrm{ml}$, respectively; $\mathrm{P}<0.05$ ). Patients who underwent laparoscopic colectomy had marginally higher overall hospitalization costs compared with the patients from the open group ( $¥ 56,484$ vs. $¥ 56,161$, respectively; $\mathrm{P}<0.05$ ). The post-operative rates of wound infection and abdominal abscess were lower in the laparoscopic group compared with the open group (17 vs. $31 \%$; and 6 vs. $18 \%$, respectively; both $\mathrm{P}<0.05)$ and no significant differences in additional post-operative complications were identified between the two groups. 
Table V. Pathological characteristics.

\begin{tabular}{lccc}
\hline Characteristics & Open & Laparoscopic & P-value \\
\hline T, n & & & 0.213 \\
$1-2$ & 15 & 15 & \\
$3-4$ & 87 & 49 & \\
$\mathrm{~N}, \mathrm{n}$ & & & 0.312 \\
- & 69 & 48 & \\
+ & 33 & 16 & \\
No. of lymph nodes & $11.81 \pm 5.40$ & $11.68 \pm 5.69$ & 0.921 \\
harvested & & & \\
TNM stage, n & & & \\
I & 14 & 13 & \\
II & 55 & 35 & \\
III & 33 & 16 & \\
\hline
\end{tabular}

$\mathrm{T}$, size and/or extension of primary tumor; $\mathrm{N}$, regional lymph nodes. Data are presented as mean \pm standard deviation unless otherwise stated.

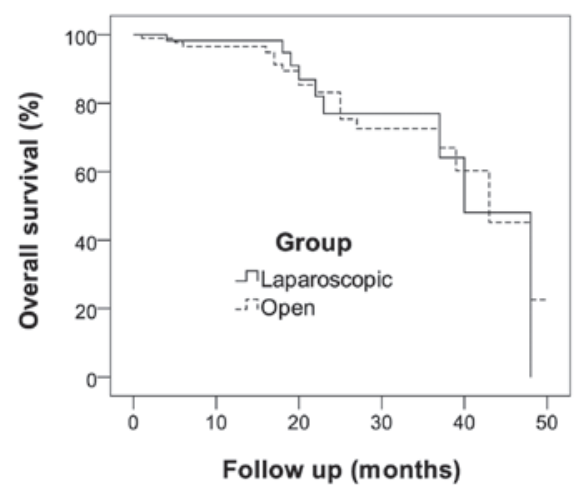

Figure 1. Overall survival rate of laparoscopic and open colectomy in colorectal cancer (CRC) patients with a BMI $>28$. Kaplan-Meier analysis (log-rank test, $\mathrm{P}=0.85$ ).

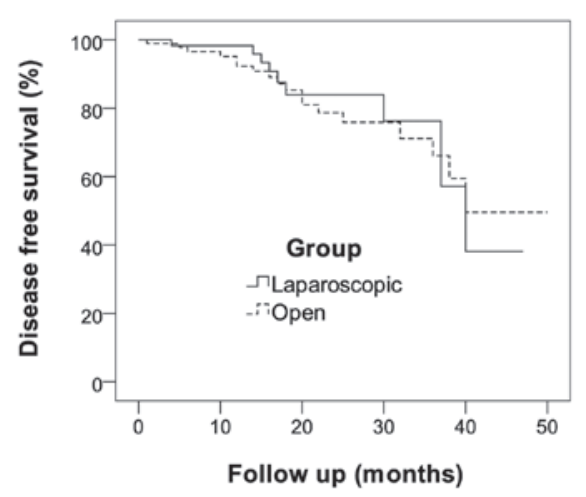

Figure 2. Disease-free survival rate of laparoscopic and open colectomy in colorectal cancer $(\mathrm{CRC})$ patients with a BMI $>28$. Kaplan-Meier analysis (log-rank test, $\mathrm{P}=0.76$ ).

As demonstrated in Table III, conversion to open surgery was required in 3 cases (4.7\%) from the laparoscopic group due to severe intra-abdominal adhesion, obesity-hindering vision and bleeding. In addition, there was one repeat surgery case in the laparoscopic group due to a small bowel obstruction and two cases in the open group due to anastomotic hemorrhage and leakage (Table IV). An additional patient with comorbidity of cardiac insufficiency developed acute heart failure and succumbed to cardiac complications on day 28 following open radical resection of sigmoid colon cancer. No significant differences in pathological characteristics were identified between the two groups, as shown in Table V.

Patients were followed until mortality or for 1-50 months. The median follow-up period was 17 months by means of outpatient services, phone calls and mail/e-mail. At the end-point of follow-up, the survival rate was $133 / 166$ patients (80.1\%). Local recurrence was identified in 14 patients $(8.8 \%)$ and 34 patients developed distal metastases during follow-up, including 20 hepatic, 8 pulmonary, 4 hepatopulmonary, 1 osseous and 1 brain metastasis. During the follow-up period, tumor-related mortalities occurred in 33 patients (19.9\%). The overall survival time in the laparoscopic and open groups was $38.7 \pm 2.7$ and $38.7 \pm 2.0$ months, respectively (Fig. 1) and the disease-free survival time was $37.3 \pm 2.5$ and $38.7 \pm 2.0$ months, respectively (Fig. 2). Results of the log-rank test demonstrated that the overall and disease-free survival rates for the laparoscopic and open groups were equivalent.

\section{Discussion}

A number of previous studies have shown that laparoscopic colectomy is technically and oncologically safe $(7,8)$ with reduced invasive manipulation and improved intra-abdominal vision and post-operative recovery compared with traditional open surgery. Due to limitations in the technique, instruments and surgical experience, obese individuals were not previously treated using laparoscopic colectomy. The increased levels of fat tissue in obese patients affects surgery by hindering visualization, dissection of the tissue planes and ligation of the vessels (9). Due to increasing surgical experience, a previous study reported the optimistic outcome of practicing laparoscopic colectomy on obese patients, although the surgery continues to be technically difficult (6). Therefore, with surgical safety and oncological clearance guaranteed, the application of a laparoscopic technique for colectomy in obese patients is promising.

BMI converts obesity into a numerical concept and is commonly used to define the level of obesity in patients. In accordance with the classification of the WHO, obesity is defined as a BMI $>30 \mathrm{~kg} / \mathrm{m}^{2}$ and this figure is widely accepted in Western countries (1). However, in China, the criteria for obesity is lower with a BMI $>28 \mathrm{~kg} / \mathrm{m}^{2}$ (2) due to the analysis of data collected via a large census of the Chinese population in the 1990s. In addition, other results have revealed that Asian Pacific populations, including that of China, have an elevated risk for obesity-related diseases at a lower BMI when compared with that of Caucasians (10). Therefore, the Chinese criteria for obesity, BMI $>28$, was adopted for the present study (11).

Previous studies have identified that obesity functions as an independent factor leading to a poorer short-term surgical outcome and long-term prognosis (12). In laparoscopic and open surgery, obesity correlates with an increased pre-operative comorbidity, volume of blood loss, surgical difficulty 
and post-operative complications. In addition, obesity shows a significantly higher rate of conversion to open surgery when compared with normal BMI patients (4). Obese individuals represent a unique subset in the treatment of CRC since obese patients are likely to suffer from increased operative difficulties, conversion rates and post-operative morbidities. These negative factors are capable of counteracting the advantages of undergoing laparoscopic surgery. Therefore, the decision between laparoscopic colectomy and open surgery for obese patients in accordance to an analogy of short-term surgical outcome in the normal population is not reliable. Few clinical studies have analyzed the short-term surgical outcomes between laparoscopic and open colectomy in obese patients, therefore, the aim of the current study was to investigate the surgical outcome of laparoscopic colectomy for CRC in obese patients. The results are likely to improve the selection of a suitable surgical approach for this subset of patients.

A previous study compared the short-term surgical outcomes of laparoscopic and open colorectal surgery in the population as a whole, including obese patients, and indicated that the laparoscopic technique is associated with a prolonged surgical duration but decreased intraoperative blood loss (13). These results are similar to those of the current study, which focused on obese patients, as when compared with the open group, the laparoscopic group also showed a significantly higher mean duration of surgery (183 vs. 167 min; $\mathrm{P}<0.05)$ and decreased blood loss (168 vs. $188 \mathrm{ml}$, respectively; $\mathrm{P}=0.02$ ). Similarly, a previous study by Balentine et al analyzed the records of 155 obese patients (BMI >30) and showed decreased intraoperative blood loss and a higher surgical duration in the laparoscopic group compared with the open group (14). Previous studies have identified that the increased difficulty of mesentery dissection and main vessel ligation in obese patients contributes to a prolonged surgical duration (15). These difficulties in manipulation are increased in obese patients due to restricted intra-abdominal space for surgery and a fatty mesentery (15). Decreased blood loss was observed in the laparoscopic group of the current study when compared with that of the open group (168 vs. $188 \mathrm{ml}$, respectively; $\mathrm{P}=0.02$ ); this is likely to be due to the ability to magnify the field of view under the laparoscope. Adjusting the angle of the $30^{\circ}$ laparoscope may improve the surgeon's vision in a comfortable position allowing dissection and ligation with increased precision. This reduces the risk of main vessel injury. Mobilization of the intestines was avoided due to the satisfactory conditions of the anatomy of the surgical region. However, despite a significant difference in the mean blood loss $(20 \mathrm{ml})$, the decreased volume recorded in the laparoscopic group resulted in no differences in the requirements for transfusion. Another study has shown that a decreased requirement for transfusion may correlate with increased post-operative morbidity (16).

In addition, no significant differences between the number of days to the first bowel movement, initiation of fluid diet, indwelling drainage and hospitalization were identified between the laparoscopic and open groups, despite slight decreases observed in the laparoscopic group. However, the rate of wound infection was significantly decreased in the laparoscopic group compared with the open group (17 vs. $31 \%$, respectively; $\mathrm{P}=0.047)$. The increased thickness of the adipose tissue layer in obese patients usually leads to a higher incidence rate of fat liquefaction, incision infection and impaired wound healing (17). Methods, including drainage and changing the dressing of the wound, are required to control infection and therefore the length and cost of hospitalization increases. Previous studies have identified that the length of the incision correlates positively with the risk of wound infection. The retractor used in open surgery exposes the surgical field by separating the incision edges. However, this is capable of inducing hypoxia at the incision site, which increases the risk of wound infection (18). In laparoscopic surgery, a pneumoperitoneum is used to expose the surgical field instead of a retractor. A pneumoperitoneum spreads the pressure on the abdominal wall and subsequently reduces the risk of hypoxia in the tissue compared with open surgery. A shorter incision is made to remove the resected tumor or perform anastomosis in specific types of laparoscopic colectomy, and in addition, the incision may be eliminated completely in specific anterior resections via a pull-through technique (19). The significantly decreased rate of wound infection following laparoscopic colectomy may be due to decreased pressure on the incision and a shorter incision length.

Abdominal abscesses are a serious complication following surgical practice and the complex nature of a number of CRC cases of these lesions may lead to their formation. Diagnosis and treatment is often delayed due to atypical clinical signs (20). The decreased incidence of abdominal abscesses in the laparoscopic group compared with the open group (6 vs. 18\%, respectively; $\mathrm{P}=0.037$ ) observed in the current study has not been previously reported (14). Anastomotic leakage has previously been confirmed as the most common cause of abdominal abscesses, however, the results of the present study showed no significant differences between the incidence of abscess and this cause (21). Therefore, it is hypothesized that the decreased incidence of abdominal abscess results from reduced mobilization and dissection of the mesentery during laparoscopic surgery compared with open surgery, and this is likely to aid the prevention of injury to fat tissue and abscess formation.

Hospitalization costs in the present study were defined as the total expenses between admission and discharge, including the costs of surgery, examination and the treatment of complications. The average hospitalization cost in the laparoscopic group was $¥ 56,484$, which was an additional $¥ 323$ compared with the cost in the open group $(\mathrm{P}<0.05)$. In the early development of laparoscopic colectomy, overall costs were reported to be higher than that of traditional open surgery (22). A prospective cost analysis in the UK compared the costs of laparoscopic and open colorectal surgery and revealed that surgery costs were higher in laparoscopic surgery when compared with that of open surgery ( $£ 2,049$ vs. $£ 1,263$, respectively; $\mathrm{P}<0.001$ ), however, significantly lower hospitalization costs following laparoscopic surgery ( $£ 1,807$, vs. $£ 3,468$, respectively; $\mathrm{P}<0.001$ ) were also identified. Therefore, the overall costs were almost equivalent ( $£ 3,875$ vs. $£ 4,383 ; \mathrm{P}=0.308)(23)$. In addition, a previous study performed by the Cleveland Center indicated that no significant differences were identified between the overall costs for laparoscopic and open surgery ( $\$ 4,003$ vs. $\$ 4,037$, respectively; $\mathrm{P}=0.14$ ) (24). However, in the current study, a significant difference was identified between the overall hospitalization costs in laparoscopic and open surgery, however, the $¥ 323$ difference was affordable for the majority of patients. 
The use of disposable instruments contributes to the increased surgical costs of laparoscopic colectomy compared with open surgery. However, the advantages of laparoscopic surgery in the post-operative period include reduced complications and a faster recovery. Therefore, the costs of hospitalization and mortality treatment may be decreased. The current study identified higher rates of pre- and post-operative complications in obese patients, increasing overall hospitalization cost. The rates of post-operative wound infection and abdominal abscess formation were decreased in the laparoscopic group. This was hypothesized to reduce the requirement for antibiotics and additional supportive treatment and therefore improve the cost gap between laparoscopic and open colectomy. A similar overall cost and an improved short-term outcome indicates a promising future for laparoscopic colectomy in obese CRC patients.

The post-operative pathological characteristics were similar in the two groups of the present study and no significant differences were identified between the number of harvested lymph nodes, consistent with previous studies $(8,25)$. In addition, no significant differences between overall and disease-free survival were identified during the follow-up period between the two groups. These results indicate that laparoscopic surgery does not affect survival rate and recurrence, consistent with results of a study performed by Nelson et al (26). A number of studies have reported higher survival rates at specific stages of CRC following a laparoscopic procedure compared with open surgery $(13,27)$. At present, it is widely accepted that the long-term oncological outcome for laparoscopic CRC resection is not inferior to the traditional open surgery approach, and the results of the present study identified this in patients with a $\mathrm{BMI}>28$.

To conclude, the results of the present study indicate that laparoscopic colectomy is technically and oncologically safe for treating obese CRC patients, and may represent a promising choice of surgery in clinical practice.

\section{Acknowledgements}

This study was supported by the National Natural Science Foundation of China (grant no. 81201618).

\section{References}

1. No authors listed: Obesity: preventing and managing the global epidemic. Report of a WHO consultation. World Health Organ Tech Rep Ser 894: 1-253, 2000.

2. Wang Y,Mi J, Shan XY, et al: Is China facing an obesity epidemic and the consequences? The trends in obesity and chronic disease in China. Int J Obes (Lond) 31: 177-188, 2007.

3. Patel SS, Patel MS, Mahanti S, et al: Laparoscopic versus open colon resections in California: a cross-sectional analysis. Am Surg 78: 1063-1065, 2012.

4. Gendall KA, Raniga S, Kennedy R and Frizelle FA: The impact of obesity on outcome after major colorectal surgery. Dis Colon Rectum 50: 2223-2237, 2007.

5. Singh A, Muthukumarasamy G, Pawa N, et al: Laparoscopic colorectal cancer surgery in obese patients. Colorectal Dis 13 $878-883,2011$
6. Lascano CA, Kaidar-Person O, Szomstein S, et al: Challenges of laparoscopic colectomy in the obese patient: a review. Am J Surg 192: 357-365, 2006.

7. Khan JS, Hemandas AK, Flashman KG, et al: Clinical outcome of laparoscopic and open colectomy for right colonic carcinoma. Ann R Coll Surg Engl 93: 603-607, 2011.

8. Leroy J, Ananian P, Rubino F, et al: The impact of obesity on technical feasibility and postoperative outcomes of laparoscopic left colectomy. Ann Surg 241: 69-76, 2005.

9. Gervaz P, Pikarsky A, Utech M, et al: Converted laparoscopic colorectal surgery. Surg Endosc 15: 827-832, 2001.

10. WHO Expert Consultation: Appropriate body-mass index for Asian populations and its implications for policy and intervention strategies. Lancet 363: 157-163, 2004.

11. Zheng W, McLerran DF, Rolland B, et al: Association between body-mass index and risk of death in more than 1 million Asians. N Engl J Med 364: 719-729, 2011.

12. Khoury W, Kiran RP, Jessie T, et al: Is the laparoscopic approach to colectomy safe for the morbidly obese? Surg Endosc 24: 1336-1340, 2010.

13. Law WL, Poon JTC, Fan JKM and Lo OS: Survival following laparoscopic versus open resection for colorectal cancer. Int $\mathbf{J}$ Colorectal Dis 27: 1077-1085, 2012.

14. Balentine CJ, Marshall C, Robinson C, et al: Obese patients benefit from minimally invasive colorectal cancer surgery. J Surg Res 163: 29-34, 2010.

15. Hotta T, Takifuji K, Yokoyama S, et al: The impact of obesity on learning laparoscopic surgery for colon cancer. J Laparoendosc Adv Surg Tech A 22: 635-640, 2012.

16. Poulsen $M$ and Ovesen H: Is laparoscopic colorectal cancer surgery in obese patients associated with an increased risk? Short-term results from a single center study of 425 patients. J Gastrointest Surg 16: 1554-1558, 2012.

17. Orcutt ST, Balentine CJ, Marshall CL, et al: Use of a Pfannenstiel incision in minimally invasive colorectal cancer surgery is associated with a lower risk of wound complications. Tech Coloproctol 16: 127-132, 2012.

18. Greif R, Akça O, Horn EP, et al; Outcomes Research Group: Supplemental perioperative oxygen to reduce the incidence of surgical wound infection. N Engl J Med 342: 161-167, 2000.

19. D'Hoore A and Wolthuis AM: Laparoscopic low anterior resection and transanal pull-through for low rectal cancer: a Natural Orifice Specimen Extraction (NOSE) technique. Colorectal Dis 13 (Suppl 7): 28-31, 2011

20. Phitayakorn R, Delaney CP, Reynolds HL, et al; International Anastomotic Leak Study Group: Standardized algorithms for management of anastomotic leaks and related abdominal and pelvic abscesses after colorectal surgery. World J Surg 32: 1147-1156, 2008

21. Eberhardt JM, Kiran RP and Lavery IC: The impact of anastomotic leak and intra-abdominal abscess on cancer-related outcomes after resection for colorectal cancer: a case control study. Dis Colon Rectum 52: 380-386, 2009.

22. Janson M, Björholt I, Carlsson P, et al: Randomized clinical trial of the costs of open and laparoscopic surgery for colonic cancer. Br J Surg 91: 409-417, 2004.

23. Dowson HM, Gage H, Jackson D, et al: Laparoscopic and open colorectal surgery: a prospective cost analysis. Colorectal Dis 14: 1424-1430, 2012.

24. Delaney CP, Pokala N, Senagore AJ, et al: Is laparoscopic colectomy applicable to patients with body mass index $>30$ ? A case-matched comparative study with open colectomy. Dis Colon Rectum 48: 975-981, 2005.

25. Sakamoto K, Niwa S, Tanaka M, et al: Influence of obesity on the short-term outcome of laparoscopic colectomy for colorectal cancer. J Minim Access Surg 3: 98-103, 2007.

26. The Clinical Outcomes of Surgical Therapy Study Group: A comparison of laparoscopically assisted and open colectomy for colon cancer. N Engl J Med 350: 2050-2059, 2004.

27. Capussotti L, Massucco P, Muratore A, et al: Laparoscopy as a prognostic factor in curative resection for node positive colorectal cancer: results for a single-institution nonrandomized prospective trial. Surg Endosc 18: 1130-1135, 2004. 